\title{
Perspectives/Opinion
}

\section{La santé mentale des étudiants : mieux prendre la mesure et considérer les enjeux}

Student's mental health: better measurement and considereration of the challenges

Y. Morvan ${ }^{\mathrm{a}, \mathrm{b}, *}$, A. Frajerman ${ }^{\mathrm{c}, \mathrm{d}}$

a Université Paris Nanterre, UFR SPSE, laboratoire CLIPSYD, EA4430, Nanterre, France

${ }^{\mathrm{b}}$ Inserm U1018, CESP, Epistémologie et méthodes de la recherche en pédopsychiatrie et santé mentale des enfants et des adolescents, Paris, France

c Inserm U1266-GDR 3557, institut de psychiatrie et neurosciences de Paris, institut de Psychiatrie, Paris, France

${ }^{\mathrm{d}}$ Université de Paris, Paris, France

\section{A R T I C L E I N F O}

\section{Article history:}

Received 1 August 2020

Accepted 30 October 2020

Available online $\mathrm{xxx}$

\section{Mots clés:}

Santé mentale

Etudiants

Dépression

Méthodologie

Mesures

Keywords:

Mental health

Students

Depression

Methodology

Measures

\section{R É S U M É}

La santé mentale représente un coût élevé pour la société pourtant la prévention semble efficace et rentable. Toutefois plusieurs problèmes se posent concernant la mesure en santé mentale et notamment de définir ce que l'on entend par pathologie. Il convient également de comprendre comment les mesures de ces concepts sont opérationnalisées et comment les outils sont construits. Ainsi pour la dépression, il existe de nombreuses échelles différentes qui ne prennent pas en compte les mêmes symptômes et pour une même échelle le seuil et la période de temps étudiés peuvent varier, ce qui a des conséquences sur les données de prévalence obtenues. Il est également important de s'interroger sur les hypothèses et modèles utilisés concernant les causes des pathologies psychiques qui sous-tendent les outils et les recherches réalisées. Pour finir, les troubles mentaux, tels que définis actuellement, débutent majoritairement avant l'âge de 24 ans pour trois quarts des personnes. Les étudiants, dont l'âge est majoritairement compris en 18 et 25 ans, sont une population particulièrement à risque ce qui peut avoir des conséquences très importantes tant individuelles que sociales. Les conséquences du coronavirus et du confinement, en lui-même et en raison de ses conséquences économiques et sociales, pourraient avoir un impact délétère sur leur santé mentale. Malheureusement, il existe encore peu de données sur les étudiants français et les études existantes ne sont pas facilement comparables en raison de méthodologies différentes. Tous ces éléments plaident pour la mise en place d'une politique d'évaluation de la santé mentale des étudiants.

A B S T R A C T

Mental health represents a high cost for society, estimated at 109 billion euros per year in France, $80 \%$ of which are indirect costs. Mental diseases start before the age of 24 for $75 \%$ of patients. Students, whose age is predominantly between 18 and 25 , are a particularly high-risk population and for whom mental illness can have very serious consequences. The Observatoire de la vie Etudiante surveys from 2016 found a 12 month prevalence of depression of $15 \%$ with suicidal thoughts in $8 \%$ of the students higher than what is observed in the French general population, respectively $10 \%$ and $5 \%$. The confinement itself and its consequences both economic (unemployment, difficulty for young people to find a job...) and social (isolation) could have a very bad effect on their mental health. The survey made by a governmental organization (Santé Publique France) revealed a significant increase in the prevalence of anxiety disorders at the start of confinement in the general population. This prevalence decreased during confinement but remained significantly higher than in 2017. Economic simulated data indicate that prevention in mental health could not only be effective but also profitable. In France, reimbursement by national health insurance of 12 sessions of psychologists for young people (between 11 to 21 ) is being tested in order to further and widely implement psychological prevention strategies rather than relying on already widely reimbursed pharmacological treatments. There are, however, several issues to discuss. First of all, is the need to define what psychopathology is considered to be. Then, it should be understood how measures of these concepts are created, how tools are constructed and how they operate in their environment. For depression, many different scales exist and even if the most used one are taken into account, they have very little content or symptoms in common. In addition, for the same scale, many different cut-offs exist to define whether a case should be considered as pathological or not, and

\footnotetext{
* Auteur correspondant.

Email address: ymorvan@parisnanterre.fr (Y. Morvan)
} 
so it is with the period of time studied being considered that may vary, both leading to consequences such as the differences observed in prevalence. Other biases should also be considered such as the age of the participants, the gender, the size of the sample, the response rate, the method of assessment and recruitment. Finally, it is necessary to question the assumptions and models used concerning the causes of psychic pathologies. Biological hypotheses on the origin of depression involve genetics and inflammation, but sociological and psychological factors must also be considered as well as the underlying complexity both in their nature and interactions at different structural levels of space and time. We should avoid drifting towards a biological or a sociological reductionism and move forward through complex systems approaches and models. With regard to student mental health in France, unfortunately, quality data are still lacking, and existing studies are difficult to compare as some may also have methodological issues. This article leads to the conclusion that there is a need for policies to assess student mental health at both local and national levels with a reflexional thinking on the tools and scales to use as measurements of these phenomena. This approach does not require being too assertive, but should have full transparency on the way the measures were designed and obtained. Measures are as much needed as are the needs for coordinated prevention and care in mental health.

\section{Introduction}

La France sort d'une période de confinement et si les inquiétudes demeurent quant à l'évolution de l'épidémie, une autre inquiétude se fait également ressentir chez les professionnels de la santé mentale. Elle concerne les conséquences, actuelles et à venir, tant chez les personnes qui souffrent déjà de troubles psychiatriques que pour les personnes potentiellement vulnérables à l'émergence de ces troubles. La crainte d'une seconde vague de troubles psychiques à venir préoccupe autant les praticiens que les chercheurs et notamment une potentielle augmentation du risque suicidaire à venir impactant plus fortement la population des jeunes adultes. En ce qui concerne les troubles psychiatriques chez les jeunes, les étudiants constituent une population particulièrement à risque dans la mesure où les trois quarts des premiers épisodes psychiatriques semblent débuter avant l'âge de 24 ans [1] et qu'à 18 ans $15 \%$ de la population aurait déjà présenté au moins un trouble [2]. Et il convient de rappeler que, malheureusement, le suicide représentait en 2016 la deuxième cause de décès en France chez les 15-24 ans, après les accidents de la route ${ }^{1}$.

Si la santé mentale représente un coût élevé pour la société et que la prévention semble efficace et rentable comme nous allons le présenter, toutefois plusieurs problèmes se posent concernant la mesure en santé mentale que nous allons aborder dans par la suite. En premier lieu nous questionnerons la définition et ce que l'on entend par pathologie dans le champ de la santé mentale. Nous tenterons ensuite de comprendre comment les mesures de ces concepts sont opérationnalisées, comment les outils sont construits et comment ils opèrent dans leur environnement. Ainsi, en nous appuyant principalement sur les mesures du mal-être, de la détresse psychologique, de la dépression et de l'anxiété, nous chercherons à montrer quelles en sont les conséquences sur les données de prévalence obtenues et qui sont présentées, tant dans les travaux de recherche qu'auprès du grand public, notamment par la presse. Ce faisant, nous chercherons également à interroger sur les hypothèses et modèles utilisés concernant les causes des pathologies psychiques qui sous-tendent les outils et les recherches réalisées et appellerons à une meilleure prise en compte de la complexité des phénomènes considérés. Pour finir, nous questionnerons les données disponibles concernant la santé mentale des étudiants français comme la production de données et d'indicateurs les concernant. Nous conclurons sur le besoin de politiques d'évaluations de la santé mentale des étudiants, non dans une démarche trop prescriptive, mais transparente, nécessaire tant à l'évaluation des actions de prévention que des prises en charge sans, pour autant, en réduire la complexité.

\footnotetext{
${ }^{1}$ https://www.insee.fr/fr/statistiques/2386052\#tableau-figure2
}

À quel point la santé mentale est-elle importante du point de vue économique?

Un point souvent méconnu ou ignoré du grand public concerne le poids économique que ces troubles représentent pour la société. En France, le coût économique des troubles psychiatriques a été évalué à 19,8 milliards d'euros en 2016 pour les simples coûts directs (hospitalisations, soins médicaux et consommation de médicaments) soit $23 \%$ des dépenses de l'assurance maladie (CNAM, 2018) dont 5,3 milliards pour "les troubles névrotiques et de l'humeur". En 2016, ce sont plus de cinq millions de personnes qui ont pris un traitement psychotrope selon l'assurance maladie. En 2018, ce sont 420000 personnes qui ont été hospitalisées en psychiatrie pour un total de 19,4 millions de journées de présence à temps complet. Les coûts indirects sont difficiles à évaluer, mais beaucoup plus élevés car ils incluent les conséquences économiques qu'engendrent les troubles psychiques sur les proches, l'entourage, le travail ou encore en termes de perte de chances socio-économiques. Le coût économique et social des troubles psychiques était ainsi évalué à 109 milliards d'euros par an en France en $2018^{2}$.

Il existe peu de données sur les étudiants en France. Les chiffres de l'Assurance maladie par tranche d'âge donnent pour les 15 à 34 ans : 324000 personnes souffrant de troubles psychiatriques dont 137 000 "troubles névrotiques et de l'humeur" et 486000 sujets sous psychotropes dont 324000 hors pathologie psychiatrique ${ }^{3}$. Malheureusement il n'existe pas, à notre connaissance, de données spécifiques aux étudiants. Dans son enquête de 2014, la mutuelle étudiante LMDE indiquait que seulement $4 \%$ des étudiants avaient consulté un psychiatre dans les douze derniers mois, mais ne donnait pas d'informations sur le coût des soins psychiatriques chez les étudiants. Il ne semble pas y avoir d'informations plus précises dans l'enquête LMDE de 2019 ou dans les enquêtes du réseau EMEVIA. Les résultats de l'enquête Santé de l'Observatoire Nationale de la Vie Etudiante (OVE) réalisée en 2016 montrent que si près de $15 \%$ des étudiants ont souffert "d'une dépression caractérisée" dans l'année, seulement $53 \%$ ont eu recours aux soins pour ces difficultés, contre 8 à $10 \%$ de la population générale française ayant souffert des mêmes difficultés dont $68 \%$ avaient eu recours aux soins pour leurs difficultés ${ }^{4}$. Les seules autres données françaises hors OVE, à notre connaissance, proviennent d'une étude réalisée en 2005-2006 sur 964 étudiants de

\footnotetext{
${ }^{2}$ https://solidarites-sante.gouv.fr/actualites/presse/dossiers-de-presse/article/ feuille-de-route-sante-mentale-et-psychiatrie-jeudi-28-juin-2018

${ }^{3}$ https://www.ameli.fr/l-assurance-maladie/statistiques-et-publications/ etudes-en-sante-publique/cartographie-des-pathologies-et-des-depenses/ effectifs-par-age-sexe-region-et-departement/ effectifs-par-pathologie-et-par-age-selon-le-sexe.php

${ }^{4}$ http://www.ove-national.education.fr/publication/la-sante-des-etudiants/
} 
six universités du sud-est de la France et qui retrouve une prévalence "de dépression caractérisée" dans l'année de $9 \%$ mais également de $15 \%$ de "troubles anxieux" et de $8 \%$ de "troubles d'usage de substances". Le recours aux soins chez les étudiants présentant au moins un de ces trois troubles était seulement de 30,5\% [3].

\section{Quel impact potentiel de la prévention en santé mentale ?}

Selon un rapport réalisé en 2013 par l'agence européenne pour la sécurité et la santé au travail qui s'est intéressée à la rentabilité de différents types d'interventions axées sur la promotion de la santé mentale et la prévention des troubles mentaux en milieu de travail : "Les résultats [..] montrent que chaque euro dépensé dans le programme de promotion et de prévention génère des avantages économiques nets sur une période d'un an allant jusqu'à 13,62 euros". . Un rapport canadien souligne également l'intérêt économique de la prévention en santé mentale : par exemple, la création d'équipe d'intervention rapide dans la lutte contre le suicide permettrait d'économiser $1886 €$ par patient ${ }^{6}$.

Chez les étudiants également, une politique d'amélioration de l'environnement pourrait avoir un impact important sur le risque de troubles psychiatriques. Or, pour les étudiants, un trouble psychiatrique non pris en charge peut conduire à une baisse significative des résultats universitaires et augmente le risque d'abandon des études. Cela peut également conduire à des difficultés d'intégration sociale sur le long terme telles que l'obtention ou le maintien d'un emploi [4]. Les résultats de l'enquête Santé de l'OVE en 2016 indiquent que $53 \%$ des étudiants présentant un épisode dépressif majeur et $51,5 \%$ des étudiants ayant eu des idéations suicidaires avaient validé leurs examens du premier semestre (avec ou sans les rattrapages) au moment de l'enquête contre respectivement $62,5 \%$ et $62 \%$ des étudiants n'ayant pas présenté ces types de difficultés. Par ailleurs les étudiants qui présentaient un épisode dépressif majeur ont également déclaré avoir dû s'arrêter de travailler ou d'étudier près de deux mois (en moyenne) à cause de leurs symptômes.

C'est une des raisons pour lesquelles les stratégies d'interventions précoces et de suivi soutenu sur la question psychique en milieu universitaire apparaissent, en plus d'une prévention sur la santé, aussi comme des déterminants non négligeables de la réussite. Malheureusement ces aspects semblent passer trop souvent "sous le radar". . Le plan "Santé et Bien-être des jeunes" lancé fin 2016 inclut bien l'expérimentation dans huit départements du remboursement par l'assurance maladie de douze séances de psychologues pour les jeunes de 11 à 21 ans. Cela permet d'une part une prévention primaire (prévention du développement de pathologie) et secondaire (dépistage de troubles psychiatriques et orientation rapide à un psychiatre pour la mise en place d'un traitement). Mais là aussi des questions sont posées quant à sa généralisation avec des questions relatives à l'hétérogénéité voire la complexité pour les acteurs sans parler de la question de la rémunération des actes.

\section{Si prévenir c'est intervenir avant les troubles, où se situe la frontière pour le psychique?}

Lorsque l'on parle de personnes souffrant de troubles psychiatriques, il s'agit de personnes dont on s'accorde à dire qu'elles ont

\footnotetext{
5 https://osha.europa.eu/en/publications/literature_reviews/ calculating-the-cost-of-work-related-stress-and-psychosocial-risks/view

${ }^{6}$ https://www.mentalhealthcommission.ca/English/resources/mhcc-reports/ case-for-investing

${ }^{7}$ https://www.orygen.org.au/Policy/Policy-Reports/Under-the-radar
}

déjà franchi un certain seuil se reflétant par une combinaison de symptômes et un niveau de retentissement de ces symptômes sur leur fonctionnement habituel et quotidien. Ce seuil étant fixé par convention (dans les classifications diagnostiques internationales), on parlera de personnes potentiellement vulnérables à l'émergence de ces troubles ou souffrant de troubles psychiques comme d'une catégorie de personnes qui présentent certes, des signes et/ou des symptômes voire un retentissement sur leur fonctionnement, mais à un niveau qui se situe en dessous du seuil fixé par convention. C'est le cas par exemple lorsqu'elles présentent des combinaisons variables de signes ou de symptômes moins spécifiques, moins graves, moins intenses, moins fréquentes et avec un retentissement ou un impact moindre sur leur fonctionnement. Cette catégorie de "cas" ou de "population" est souvent dénommée dans la littérature scientifique avec les termes : "subsyndromique" ou encore "à risque". Lorsque l'on considère les personnes qui présentent des signes et/ou des symptômes sans tenir compte du seuil fixé par convention, c'est-à-dire à la fois les personnes "à risque" et les personnes qui pourraient potentiellement souffrir d'un trouble psychiatrique, alors cette catégorie est souvent dénommée dans la littérature scientifique avec les termes "vulnérables" et représente souvent un nombre plus important de personnes du point de vue de la population.

À titre d'illustration, la dernière enquête Santé de l'OVE en $2016^{8}$, qui tentait de mesurer la dépression, indiquait que $37 \%$ des étudiants avaient déclaré des signes de tristesse et $33 \%$ des signes d'anhédonie pendant au moins deux semaines au cours de l'année écoulée. Dans $22 \%$ des cas, ces signes étaient suffisamment intenses et fréquents, car présents quasiment tous les jours et quasiment toute la journée pendant ces deux semaines, pour être qualifiés de symptômes correspondant à une population "vulnérable". Dans $18 \%$ des cas, un nombre suffisant de signes ou de symptômes supplémentaires dits "secondaires" étaient déclarés toujours durant ces deux semaines pour correspondre aux seuils dits "cliniques" ou symptomatologiques de la dépression selon les classifications diagnostiques internationales. Ces symptômes déclarés étaient par exemple une prise ou perte de poids de plus de cinq kilogrammes en l'absence de régime, des troubles du sommeil, de l'épuisement ou un manque d'énergie plus qu'à l'habitude, des troubles de la concentration, des sentiments de dévalorisation ou des pensées liées à la mort. Dans $15 \%$ des cas, cette combinaison de signes ou de symptômes s'accompagnait d'un retentissement suffisamment important sur le fonctionnement des étudiants par rapport à l'habitude correspondant à un épisode dépressif dit caractérisé ou majeur toujours selon ces mêmes classifications. Enfin, ce sont $5 \%$ des étudiants qui se situaient à l'extrémité du seuil de gravité des troubles psychiatriques en déclarant un nombre important de symptômes avec un retentissement élevé correspondant à un épisode dépressif pouvant être considéré comme sévère et nécessitant probablement une hospitalisation dans un service spécialisé.

\section{La pathologie psychique, un concept flou : trouble ou maladie?}

Une des difficultés « fondatrices » concernant la santé psychique et notamment sur son versant pathologique repose sur la conceptualisation théorique, les hypothèses ainsi que les opérationnalisations telles que les définitions mêmes des objets étudiés. Si les termes de «santé mentale, mal-être, dépression, troubles mentaux» ne semblent pas poser de problème de définition a priori, il semble pourtant comme nous l'avons illustré avec le mal-être des étudiants que la plupart de ceux qui utilisent ces termes «s'autorisent l'illusion confort-

\footnotetext{
${ }^{8} \mathrm{http} / /$ www.ove-national.education.fr/publication/la-sante-des-etudiants/
} 
able que tout le monde comprend de quoi l'on parle » [5]. Il faut pourtant rappeler que pour la question du psychique considéré comme un état de santé, il n'existe pas de signes directement observables ou du moins suffisamment spécifiques qui permettraient d'établir une base consensuelle pour le diagnostic. De manière générale, les classifications diagnostiques des troubles ont donc été établies sur la base de groupements de symptômes observables. Nous avons illustré avec les résultats de l'enquête de l'OVE à quel point le critère de seuil retenu pouvait varier en reposant sur des choix différents relativement au nombre de signes ou de symptômes, de leur intensité, fréquence et du degré de retentissement sur le fonctionnement habituel considéré pour définir la mesure choisie. Ces choix ont des incidences directes sur la fréquence du trouble que l'on cherche à mesurer et donc sur la manière dont ils sont recueillis et peuvent être interprétés. Connaître la manière précise dont sont recueillies les données relatives à la santé psychique dans les enquêtes constitue donc un enjeu majeur. En effet, cela permet de mieux comprendre comment les différents signes et symptômes interrogés dans une enquête sont censés représenter l'étiquette diagnostique apparemment « uniforme » par exemple d'un trouble comme la dépression.

Mais d'ailleurs pourquoi parlons-nous de troubles et pas de maladie ? Contrairement à l'intuition, cela n'a en réalité pas de lien direct avec le degré de souffrance des personnes touchées ni le degré d'impact personnel, pour les proches où la société qu'impliquent ces difficultés. En médecine, il est envisageable d'utiliser le terme de maladie si les manifestations symptomatologiques que l'on observe ont en réalité une cause commune autrement appelée "étiologie". Un exemple célèbre de l'histoire médicale concerne le syndrome de paralysie générale comprenant diverses manifestations symptomatiques d'ordre neuropsychiatrique. De nombreux débats ont ainsi animé la communauté scientifique et médicale au $\mathrm{XIX}^{\mathrm{e}}$ siècle sur la classification et l'origine de ces troubles avec l'hypothèse d'une cause neurologique commune aux différents tableaux cliniques observés et des différents cours évolutifs temporels de ces tableaux. Ce modèle de la cause commune ayant pu être finalement démontré par l'identification de la présence de la bactérie Treponema pallidum dans le cerveau des patients atteints et confirmé par l'efficacité d'un antibiotique, la pénicilline, comme traitement de cette étiologie. Or, si l'on parle de troubles et non de maladies pour les pathologies psychiques c'est que justement aucune cause commune ou étiologie n'a encore pu être démontrée pour l'ensemble de ces pathologies.

Si la recherche se poursuit notamment en génétique, biologie, pharmacologie, immunologie et imagerie pour tenter d'identifier les étiologies de différents troubles, cette recherche, en plus des limites méthodologiques techniques et technologiques actuelles, se heurte également aux écueils liés à la définition et aux hypothèses quant à la nature des troubles et à leurs mesures. Elle se limite également par une forme de réductionnisme se risquant à une biologisation de la psychopathologie. En guise de conclusion d'une revue autobiographique de ses travaux de recherche sur les facteurs de risque de la dépression, Constance Hammen précisait ce que l'on pouvait entendre derrière ce risque de réductionnisme dans la mesure où les facteurs biologiques semblent bien impliqués dans les processus étiologiques et constituent une importante voie de recherche : « Cela a abouti, parfois, à un langage bâclé ou à une croyance profondément enracinée dans l'existence de bases biologiques ou de causes sous-jacentes du trouble, attribuées aux neurones, à des structures cérébrales, à des propriétés chimiques, ou à des caractéristiques génétiques. Certaines des formes de réductionnisme (par exemple, le réductionnisme éliminatoire) présument la primauté du pouvoir explicatif des niveaux inférieurs de description (biologique) sur les niveaux supérieurs, y compris la description psychologique ».
Elle précisait également les opportunités à venir du point de vue de la recherche dans une perspective de prise en compte de la complexité des phénomènes que nous tentons de mesurer et d'expliquer : "Les progrès de nos connaissances sur le cerveau et la génétique et leurs relations avec le comportement et les émotions sont indéniablement excitants et d'une importance vitale, mais ils ne sont qu'une partie de la chaîne causale des événements, pas la fin. J'attends avec impatience les développements scientifiques et cliniques dans les années à venir et souhaite sincèrement à tous les jeunes chercheurs qui étudient la dépression de ressentir l'excitation, les défis de croissance et le sens du progrès dont j'ai eu la chance d'être témoin au cours des dernières décennies » [6]. Une récente rétrospective sur les 50 dernières années concernant la prévention et la prise en charge de la dépression met également en lumière une forme de complexité autour des effets différenciés de l'action des psychothérapies et des antidépresseurs sur le cerveau dans ce cadre [7].

\section{Pourquoi est-il si important de bien définir nos termes? Le cas du mal-être étudiant}

Bien définir le sujet constitue souvent une première difficulté en soi et, "au-delà de l'illusion confortable", qu' entend-on réellement par santé mentale : dépression, idées suicidaires, suicide, souffrance psychologique, stress, mal-être ? Que ce soit pour le grand public et les décideurs politiques, la problématique d'exposition et de compréhension des termes employés à travers les différents rapports qui sont réalisés et synthétisés dans la presse pose problème. Si l'on s'intéresse à la question de la santé des étudiants et plus particulièrement celui de la santé psychique, le mal-être est une problématique souvent évoquée. Prenons par exemple le cas de deux études réalisées par des mutuelles étudiantes auprès de leurs adhérents. En 2013, la mutuelle étudiante SMEREP indiquait dans son communiqué de presse que 76 $\%$ des étudiants expriment un état de mal-être conjuguant trois sentiments : un état de stress avec une perte de confiance et déprime. Ces sentiments devaient être présents pendant au moins deux semaines consécutives durant l'année écoulée. Dans une autre enquête réalisée par la mutuelle étudiante LMDE auprès de ses adhérents en 2011 et s'intéressant également au mal-être, les résultats indiquaient cette fois que $38 \%$ présentaient des signes de mal-être qui étaient définis comme le fait d'avoir ressenti un sentiment constant de détresse et de déprime au cours de l'année écoulée. Par ailleurs, l'enquête de la LMDE de $2019^{9}$ (du 28 mars au 29 avril 2019, en ligne, 5861 réponses) retrouve la présence d'idées suicidaires sur les 12 derniers mois chez $30 \%$ des étudiants tandis que cette même prévalence est de $8,4 \%$ dans l'enquête santé de l'OVE ${ }^{10}$ et de $4,7 \%$ chez les $18-75$ ans en population générale [8].

Plusieurs éléments, tels que la définition précise de la mesure et des questions directement posées aux étudiants ou encore de l'algorithme de calcul employé, peuvent, en dehors d'autres éléments comme le taux de participation ou la représentativité des données, contribuer à expliquer ces différences de résultats. Il apparaît toutefois évident que, du point de vue du lecteur quel qu'il soit, annoncer à un moment qu'un tiers des étudiants souffrent de mal-être puis d'annoncer ultérieurement que ce sont en fait les trois quarts des étudiants qui souffrent de mal-être interroge, soit sur le sérieux de la mesure soit sur l'évolution désastreuse de la situation en l'espace de deux années seulement. Si l'on devait comparer cela à l'annonce de deux températures, il s'agirait de savoir laquelle est en degré Celsius ou Fahrenheit ou bien si nous sommes passés de l'hiver à l'été en très

\footnotetext{
${ }^{9}$ https://www.lmde.fr/commentcava

${ }^{10} \mathrm{http} / /$ www.ove-national.education.fr/publication/la-sante-des-etudiants/
} 
peu de temps. De manière moins absolue en termes d'hypothèses, il est évidemment possible d'envisager une combinaison entre effet de mesure et évolution de la situation, mais la part précise entre ces deux aspects se doit d'être évalué. C'est la raison essentielle pour laquelle nous devons faire l'effort de préciser certains termes, parfois flous, qui renvoient à des définitions et des opérationnalisations formelles différentes des mesures portant sur la santé psychique.

\section{Est-ce toujours la même dépression que l'on mesure ?}

Trois grandes difficultés sont souvent présentées comme relatives aux problématiques de validité des classifications diagnostiques existantes. La première concerne le fait que nombre de manifestations symptomatologiques sont dites comorbides, c'est-à-dire qu'elles sont présentes dans différentes catégories de troubles, et en plus que ces catégories soient très fréquemment comorbides ce qui signifie qu'elles sont souvent présentes en même temps (par exemple l'anxiété et la dépression) [9]. La seconde concerne le fait qu'il puisse tout simplement ne pas y avoir in fine de causes communes aux troubles psychiques et que ces derniers seraient "massivement multifactoriels" et constitueraient plutôt des propriétés émergentes impliquant des systèmes complexes d'interactions entre différentes dimensions bio-psycho-sociales [10] sans compter que ces interactions peuvent également se produire sur différents niveaux temporels et spatiaux [11]. Ces modèles théoriques constituent des hypothèses alternatives au modèle de la cause commune souvent désigné comme modèle « bio-médical » dans la mesure où ils engagent ainsi, en interaction et en complémentarité avec les facteurs biologiques, d'autres aspects : psychologiques, cognitifs, environnementaux et sociétaux. Pour autant, dans le champ de la psychologie, ces modèles doivent encore et davantage s'appuyer sur la construction rigoureuse de théories et la confrontation aux données des modèles générés par ces dernières [12].

La troisième difficulté concerne la comparabilité des données en lien avec la très grande hétérogénéité des outils que l'on utilise pour mesurer un phénomène. En effet, il existe un grand nombre d'échelles différentes pour chaque indicateur. Le mal-être, la qualité de vie ou le stress sont des mesures qualitatives difficilement comparables malgré le fait qu'elles puissent entretenir des relations entre elles. À nouveau, illustrons ce point avec la dépression. Parmi 280 instruments de mesure des symptômes dépressifs recensés, le chercheur Eiko Fried a analysé sept échelles parmi les plus utilisées pour évaluer l'efficacité des traitements psychotropes ou des prises en charge psychothérapeutiques. Cette analyse très fine lui a permis d'identifier que ces sept échelles, qui représentaient 125 questions différentes, mesuraient en réalité 52 symptômes différents dont seuls $12 \%$ étaient communs aux sept échelles et $40 \%$ d'entre eux apparaissent dans une seule des sept échelles [13]. Au-delà des problématiques que cette hétérogénéité soulève, elle pourrait être expliquée par la variabilité des modèles théoriques sous-jacents et donc justifier d'une certaine manière cette faible cohérence de contenu entre ces échelles. On pourrait dans ce cas considérer qu'une définition ou un standard partagé au niveau international comme des critères symptomatologiques des classifications diagnostiques, que nous avons explicité ci-dessus, serait une solution à cette difficulté. Il semble que cela ne soit malheureusement pas le cas et que même dans le cas des outils les plus fréquemment utilisés pour évaluer les thérapeutiques nous ne devions choisir en réalité entre sept thermomètres assez différents. Eiko Fried a récemment montré que malgré l'utilisation des critères du DSM5 comprenant neuf symptômes consensuels, on pouvait aboutir en réalité à plus de 10377 profils symptomatologiques différents du fait de différence de considérations qualitatives pour chacun de ces neuf critères [14].

\section{Avec des biais de recueils et de représentativité en plus ?}

La quatrième difficulté concerne toujours la comparabilité, mais en lien, cette fois, avec l'hétérogénéité des seuils retenus, des populations étudiées et de leur représentativité. Dans une méta-analyse parue en 2016 [15] et portant sur la dépression chez les étudiants en médecine, les auteurs ont compilé des données provenant de 193 études différentes. Les méthodologies sont très diverses et concernent en réalité des populations très hétérogènes : ainsi le nombre de participants par étude varie de 43 à plus de 10 000, l'année d'étude des participants varie de la première à la sixième année (les trois premières années, les étudiants sont à l'université et les trois années suivantes en stage à l'hôpital au contact des patients), l'âge moyen des participants varie de 17,5 à 26 ans, le pourcentage d'hommes ou de femmes participants varie entre 0 et $100 \%$. Enfin les taux de réponses sont également très variables (par exemple de $35 \%$ à $89 \%$ ). Les outils de mesure de la dépression sont également très variables, car ce sont 26 échelles différentes qui ont été utilisées et avec des seuils très variés pour qualifier la présence d'une dépression ou non. À titre d'exemple, pour le score total a obtenu à l'échelle Hospital Anxiety and Depression Scale (HAD), on recense quatre seuils différents $[7,8,11,12]$ pour retenir un diagnostic de « dépression » comme «présent ». Dans cette même étude, on retrouve également cinq seuils différents pour la " présence » d'une " dépression » avec le score total obtenu par les participants à l'échelle Zung Self-Rating Depression Scale. Afin de mieux prendre en compte cette hétérogénéité des seuils (et implicitement l'hétérogénéité potentielle qu'elle implique vis-à-vis du trouble que cette échelle est censée représenter), les auteurs ont fait des analyses en fonction du type d'échelle et du score seuil retenu pour qualifier la présence d'une « dépression », ce qui entraîne des variations de prévalence allant de 9 à $56 \%$ en fonction de l'année d'étude (pré clinique soit de la première d'étude à la troisième année vs clinique : de la quatrième à la sixième année d'étude). Une variabilité de « dépression » entre 17 à $46 \%$ se retrouve également en fonction des différents pays où les études ont été réalisées. C'est pour cela que les auteurs, par mesure de prudence, préfèrent parler de « syndrome dépressif » plutôt que de « dépression ».

En France, l'ISNI (Inter Syndicat National des Internes) a mené deux enquêtes en $2017^{11}$ et $2020^{12}$ sur la dépression chez les internes en médecine. Elle a utilisé la HAD avec un seuil à huit pour qualifier la présence de "dépression 》 en 2017 puis de onze en 2020. Les autres limites de cette étude sont l'absence de données démographiques disponibles telles que l'âge moyen ou encore le pourcentage de femmes et d'hommes. Le taux de réponse n'ayant probablement pas pu être calculé dans la mesure où l'étude a été réalisée en ligne, l'étude ne donne pas non plus d'indications quant au taux d'abandon ou encore une indication du ratio nombre de participants par rapport au nombre d'internes en France au moment de l'enquête. Si la première étude de l'ISNI ne permettait pas de comparaison avec la population générale française (Baromètre Santé des Français) en raison de l'échelle utilisée ce n'est plus le cas dans la mesure où Santé Publique France (SPF) dans le contexte du COVID et du confinement a réalisé une enquête en 2020 qui utilisait la même échelle

\footnotetext{
${ }^{11}$ https://www.anemf.org/blog/2017/06/13/ enquete-sante-mentale-des-jeunes-medecins-2/

12 https://isni.fr/vecu-psychologique-de-lepidemie-covid/
} 
(HAD) avec le même seuil (score total au minimum de 11$)^{13}$. Toutefois la méthodologie diffère dans la mesure où l'enquête de SPF permet d'identifier le pourcentage de femmes et d'hommes, l'âge, la catégorie socio-professionnelle. De plus, l'échantillon des répondants a été redressé (par une variable de pondération) afin de projeter les réponses des participants au plus près de la population générale française. Il convient donc de rester prudent quant à la comparabilité des résultats entre ces deux études. Toutefois, les résultats de la dernière enquête de l'ISNI semblent indiquer que les internes présenteraient une prévalence de $47 \%$ pour les «troubles anxieux » et de $18 \%$ pour la « dépression ». Les prévalences respectives pour les 11 enquêtes réalisées par SPF entre le 23 mars et le 24 juin 2020 étaient, pour les 25-34 ans, de $37,5 \%{ }^{14}$ et de $23,5 \%{ }^{15}$ (valeur maximale retenue pour ces 11 enquêtes $^{16}$ ).

De même, avec l'enquête réalisée par l'OVE entre le 26 juin et le 8 juillet sur la vie d'étudiant confiné ${ }^{17}$, la prévalence de la détresse psychologique en 2020 (31\%) est comparée avec celle mesurée en $2016(21 \%)^{18}$. Dans l'enquête de 2016 , la détresse psychologique est définie comme un score inférieur ou égal à 14 à l'échelle "Mental Health Inventory » (MHI-5) sur un total de 25 et est mesurée sur les quatre dernières semaines écoulées. Or, l'enquête réalisée en 2020 en reformulant les questions pour les adapter à la période de confinement mesure en réalité la détresse psychologique sur une période deux fois plus importante de huit semaines. De plus, les pourcentages de non-réponse aux questions du MHI-5 ne sont pas présentés. En effet, la prévalence donnée est, dans ces deux cas, calculée comme une proportion relative avec : a) un numérateur représentant le nombre de personnes ayant un score supérieur à un seuil donné et b) un dénominateur qui est le nombre de personnes répondant aux questions. Dans la mesure où, pour le dénominateur, il ne s'agit pas de l'ensemble des personnes constituant l'échantillon retenu des participants à chaque l'enquête, le périmètre et la représentativité de ces dénominateurs ne sont donc pas constants vis-à-vis de leurs échantillons respectifs. La différence de prévalence observée entre les enquêtes (31\% versus 20 $\%$ ) peut donc s'expliquer de plusieurs manières :

- par une aggravation de la détresse psychologique des étudiants au cours (et en raison ?) du confinement ;

- par une différence d'effet de mesure de la période temporelle considérée :

- par un effet de consigne mentionnant explicitement la période de confinement (potentiellement chargée de représentations cognitives différentes d'une simple mention des " 4 dernières semaines ») ;

- par une différence dans les taux et profils des non-répondants.

Il est bien évidemment tout à fait envisageable que ces quatre explications potentielles agissent et interagissent de concert pour produire cette différence de prévalence, mais dans quelles mesures relatives et selon quelles interactions, on ne le sait pas.

\footnotetext{
13 https://www.lemonde.fr/les-decodeurs/article/2020/05/26/

insomnie-anxiete-la-sante-mentale-des-francais-s-est-degradee-pendant-le-confinemen

14 https://www.santepubliquefrance.fr/content/download/249173/file/

enquete_coviprev_sm_tab4a_020720.pdf

15 https://www.santepubliquefrance.fr/content/download/256351/file/ enquete_coviprev_sm_tab5a_020720.pdf

16 https://www.santepubliquefrance.fr/content/download/249168/file/ enquete_coviprev_sm_tab1_020720.pdf

$17 \mathrm{http} / / /$ www.ove-national.education.fr/wp-content/uploads/2020/07/

La-vie-de $\%$ CC $\% 81$ tudiant-confine $\%$ CC $\% 81$-enque $\%$ CC $\% 82$ te-2020.pdf

18 http://www.ove-national.education.fr/wp-content/uploads/2018/11/

Reperes_sante_etudiants_2018.pdf
}

Une étude intéressante portant également sur certaines dimensions de la détresse psychologique et de la santé mentale des étudiants a débuté aux Pays-Bas très peu de temps après l'apparition des premiers cas dans le pays. Cette étude, (non encore publiée) est intéressante et complémentaire des études comme celle de l'OVE dans la mesure où elle est de nature longitudinale et a été menée durant une période de deux semaines auprès de 80 étudiants avec un questionnaire initial de 45 minutes, puis 14 questions relatives à la santé mentale et la détresse psychologique mesurées quatre fois par jour durant les deux semaines (soit 56 points de mesure au total pour chacune de ces variables) et enfin un questionnaire de fin d'étude de 20 minutes [16]. Les résultats de cette étude sont tout aussi intéressants que les questions qu'ils posent comme le résume très bien un des chercheurs impliqués dans ce travail sur son blog ${ }^{19}$. En effet, ils n'ont pas (avec les techniques d'analyses utilisées) retrouvé de changement sur la santé mentale des étudiants voire plutôt une amélioration de celle-ci dans certains cas suggérant une forte résilience de la part des étudiants ayant participé à l'étude et durant cette période d'observation. Mais les chercheurs évoquent également d'autres explications possibles à ces résultats comme des effets non-attendus d'un sentiment plus important d'appartenance à la communauté et de relations avec les proches importants durant une période de crise. Ils évoquent également un potentiel problème de mesure lié au fait qu'ils pourraient avoir, en réalité, manqué le pic de début des difficultés de santé mentale lié à la pandémie. Ce qu'ils observeraient pourrait être en réalité non pas une amélioration, mais simplement un retour à un niveau «normal » de la santé mentale à la suite d'un fort impact initial. Une dernière explication possible concerne le fait que les effets pourraient ne pas être les mêmes sur les sous-dimensions que leur outil de mesure est censé mesurer entre le début de leur étude (questionnaire initial) et la fin (questionnaire final). Ils ont, en effet, utilisé une échelle mesurant la santé mentale en 21 questions avec un score total mais cette échelle permet également de calculer des sous-scores de dépression, d'anxiété et de stress. Les auteurs indiquent que les premières analyses indiqueraient une augmentation des scores de dépression, mais une diminution des scores d'anxiété tandis que le niveau de stress ne serait pas modifié. Cependant une récente publication sur ces données suggérerait que des difficultés d'homéostasie de l'humeur (i.e. la capacité à stabiliser l'humeur au temps $t$ par le biais d'activités au temps $t+1)^{20}$ puissent être plus importantes à la suite de la crise du COVID-19 de manière générale et d'autant plus pour les personnes vulnérables ayant déjà déclaré des antécédents de troubles psychiatriques (et/ou ayant déjà pris des traitements pharmacologiques utilisés en psychiatrie au sens large) [17]. D'autres inquiétudes ont également déjà été soulevées concernant des risques psychologiques de la pandémie à plus longs termes. Ainsi, l'Association médicale australienne (AMA) a du reste alerté sur le risque d'augmentation du nombre de suicides d'ici quelques années, en tablant sur une hausse moyenne de $25 \%$, voire $30 \%$ chez les jeunes $^{21}$. Ces données, qui ne sont pas encore publiées, s'appuient sur des simulations (qu'il conviendra de valider à l'avenir) cherchant à rendre compte de l'interaction dynamique de différentes inégalités sociales, d'accès à l'emploi et aux soins avec les conséquences de la

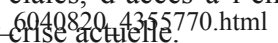

Nous avons déjà indiqué que le choix de la HAD, comme le montre la méta-analyse de Rotenstein, a ses limites en raison des varia-

\footnotetext{
19 https://eiko-fried.com/preprint-covid-19-pandemic-and-student-mental-health/

${ }^{20} \mathrm{https}$ ///technologiemedia.net/2020/04/22/

une-faible-regulation-de-lhumeur-chez-les-depressifs/

21 https://ama.com.au/media/

joint-statement-covid-19-impact-likely-lead-increased-rates-suicide-and-mental-illness
} 
tions possibles du score seuil retenu à l'échelle pour retenir la présence "d'un cas" ou plus spécifiquement ici d'un diagnostic probable de "dépression" ou "d'anxiété" [15]. Par ailleurs, dans le cas où les études sont réalisées en ligne, un article récent rappelait d'autres biais potentiels comme le fait que les personnes souffrant de troubles psychiatriques ainsi que celles ayant des difficultés économiques pourraient moins participer à ces enquêtes. La méthode de recrutement des études appelée "boule de neige" utilisée pour que les répondants participent (par exemple dans le cadre de diffusion sur des réseaux sociaux ou professionnels) pourrait également constituer un biais de sélection des participants. Dans ce cas, le biais serait plutôt dans le sens d'une sur-représentation de participants davantage concernés par le sujet de l'enquête. Ainsi, il demeure fondamental de pouvoir accéder à une description détaillée de la population, mais également de la méthodologie de constitution des échantillons d'enquêtes comme des taux de participations et/ou d'abandon pour pouvoir en évaluer tant sa représentativité que son degré de généralisabilité [18].

\section{Quelles données sur la santé mentale des étudiants actuellement en France?}

Il n'existe probablement pas suffisamment d'études et donc de données ni de mesures systématisées de la santé mentale des étudiants disponibles aujourd'hui en France. Actuellement les sources d'informations reposent principalement sur les études menées par l'OVE ou l'enquête I-Share ainsi que sur les enquêtes menées par les organisations et mutuelles étudiantes ou encore celles réalisées par diverses équipes de recherches pour des enquêtes ayant un périmètre plus local. Même si nous avons pointé certaines difficultés liées aux prévalences des « troubles », les établissements d'enseignement supérieur ne sont pas tenus de disposer d'indicateurs sur la santé mentale de leurs étudiants. Et lorsqu'ils fournissent des données, par exemple à l'occasion des procédures nationales d'évaluation les concernant, ces dernières concernent plus fréquemment le pourcentage d'étudiants ayant effectivement consulté le service de médecine préventive au décours de la « visite médicale obligatoire ». Cependant, il s'avère parfois difficile de connaître ou d'identifier facilement le dénominateur : s'agit-il des étudiants inscrits sur le cycle licence à un moment donné, du nombre cumulé sur une cohorte temporelle du cycle licence, ou seulement sur le nombre annuel de patients convoqués ou encore simplement une description de l'activité en termes de nombre de consultations ?

On pourrait également s'interroger sur les moyens dont les établissements disposent ou choisissent d'investir pour produire des indicateurs. Leur construction transparente ainsi que leur fiabilité comme la facilitation de leur production grâce au numérique posent également question. Depuis la publication en 2010 au bulletin officiel d'une circulaire concernant la médecine préventive universitaire ${ }^{22}$ : combien d'établissements impliquent leurs observatoires locaux de la vie étudiante sur les questions de santé et les mettent en appui de leurs services de médecine préventive ? Combien d'établissements disposent de logiciels d'aide et de gestion pour l'activité de médecine préventive ou leurs actions de santé qui permettent tant la production d'indicateurs que la prise de rendez-vous, l'édition de certificats et d'ordonnances et de suivi de la prise en charge ? Combien d'établissements disposent de systèmes qui automatisent la communication entre les certificats d'aménagement des études et des examens avec les services et les systèmes de scolarité ? Une thèse d'exercice de

\footnotetext{
${ }^{22}$ https://www.enseignementsup-recherche.gouv.fr/pid20536/ rubrique-bo.html?cid_bo $=51006$
}

médecine générale récente ${ }^{23}$, réalisée par le Dr Gluchowski auprès du service de santé universitaire de l'université de Lyon illustre d'ailleurs très précisément ces difficultés. Même de manière plus globale au niveau des établissements, combien disposent d'entrepôts de données permettant la production d'indicateurs par-delà même la question de leurs constructions transparentes et pérennes dans le temps ? Combien d'établissements peuvent produire des rapports automatisés et en temps réel permettant le suivi de ces indicateurs pour aider au pilotage, mais aussi et surtout pour permettre aux différents acteurs (équipe de directions, services administratifs, équipes pédagogiques jusqu' aux services universitaires en charge de la santé et du handicap) de concentrer leur temps sur leur cœur de métier ? Malheureusement là encore, une récente étude d'un cabinet d'audit montre que beaucoup de chemin reste à parcourir sur ce point ${ }^{24}$. C'est d'ailleurs dans ce cadre que nous travaillons au développement des outils numériques et applicatifs en santé étudiante ${ }^{25}$.

Si une mesure ou un indicateur a toujours des limites et peut faire débat, il n'en demeure pas moins indispensable, à la fois pour tenter de "prendre la mesure" ou de quantifier le problème. Cela s'avère nécessaire à toute tentative d'évaluation de l'efficacité des actions, que ce soit sur le plan opérationnel, de la santé ou encore dans une logique économique. Toutefois la prudence et le recul restent indispensables lorsqu'il s'agit de définir, construire puis commenter et décider sur la base d'indicateurs chiffrés. D'après les auteurs d'un commentaire paru dans la revue Nature [19], s'ils peuvent donner une apparence de rigueur, les indicateurs peuvent également donner à une décision politique l'apparence d'une décision technique. La production et l'attention excessive sur ces derniers pouvant se retourner contre le but initial du "pilotage éclairé" dans la mesure où "elle peut éloigner une discipline du grossièrement juste et l'amener au précisément faux [..] et ne peut se substituer au jugement éclairé. [..] Nous n'appelons pas à une fin de la quantification, pas plus qu'à des modèles apolitiques, mais à une transparence totale et franche". En effet, il convient d'expliciter tout aussi clairement les limites inhérentes au processus évaluatif et aux indicateurs choisis que les résultats qu'ils produisent. C'est une condition essentielle afin d'éviter que la présence d'indicateurs ne soit en réalité de véritables "chevaux de Troie pour des intérêts et des valeurs non dévoilés". Dans cette même logique, il demeure important que l'effort nécessaire de standardisation des mesures n'aboutisse pas non plus à une situation ubuesque en restreignant le champ des outils à utiliser sans considérer les " effets secondaires de ces choix », en l'occurrence leur potentielle incapacité de généralisation (s'appliquer à tout et à tous, en tous lieux et de tout temps). La confiance dans le caractère nomologique (institution d'une loi) des résultats d'études doit être notamment interrogée au regard, en psychologie, du caractère potentiellement complexe et non-ergodique des processus et phénomènes observés (hétérogénéité à travers les individus et le temps). Ainsi, les rapports ne sont pas évidents entre le fait que les résultats d'approches nomothétiques (inter-individuelles) et idiographiques (intra-individuelles) puissent être équivalents [20]. De même, dans une approche nomothétique les résultats obtenus peuvent être complètement différents si l'on s'intéresse à l'ensemble de la population observée ou si l'on s'intéresse à des groupes particuliers composant cette population. Ce phénomène est appelé le paradoxe de Simpson ${ }^{26}$. Enfin, il

\footnotetext{
${ }^{23} \mathrm{https}$ //etu.univ-lyon1.fr/guide-pratique/sante/

these-pourquoi-les-etudiants-consultent-ils-en-centre-de-sante-universite--1060175.kjsp

${ }^{24}$ https://www.ey.com/fr_fr/government-public-sector/

le-pilotage-des-etablissements-d-enseignement-superieur-a-l-heure-du-numerique

${ }^{25} \mathrm{https} / / /$ osf.io/svmzp/

${ }^{26} \mathrm{https}$ //www.outils-pour-reflechir.fr/le-paradoxe-de-simpson/
} 
est également tout aussi important de rester vigilant à bien identifier ce pour quoi les outils ont été construits et ce qu'ils peuvent " en réalité » mesurer. Ces dangers d'une culture évaluative qui serait trop prescriptrice du choix de ses outils ont été tout récemment exposés [21] et ne sont pas sans lien avec les débats qui concernent les classifications internationales comme la CIM ou le DSM et avec les alternatives que représentent les classifications RdoC et HiTOP.

\section{Ne pas réduire la complexité à la condition étudiante}

La dernière enquête de l'OVE dont les résultats sont disponibles date de 2016. Dans cette enquête, les prévalences de l'épisode dépressif majeur (EDM) et des idées suicidaires (IDS) chez les étudiants sont respectivement de $15 \%$ et $8 \%$ soit $50 \%$ d'EDM en plus comparées à la population générale et deux fois plus d'IDS. Les principaux facteurs de risque retrouvés sont les difficultés financières ressenties, les difficultés scolaires et le sexe féminin. Cette enquête utilise un outil de mesure standardisé (CIDI-SF) également utilisé dans une autre enquête nationale avec un échantillon tiré aléatoirement en population générale : le baromètre Santé des Français. Cela permet donc une comparaison entre les étudiants et la population générale. Malheureusement, une seule enquête ne permet pas d'étudier l'évolution de la santé mentale des étudiants dans le temps. Par ailleurs, les modalités de passations des enquêtes sont différentes (recours aux enquêteurs téléphoniques pour le Baromètre Santé des Français et uniquement par invitation mail et enquête web pour l'OVE). Cela peut induire également des différences comme le taux de participation et donc le degré possible de généralisation ou d'inférence des résultats à l'ensemble de la population. Une limite pour l'OVE avec notamment un taux de réponse de $19 \%$ qui est certes comparable aux données de la littérature, mais qui reste faible, même s'il correspond à 19000 participants. Une autre limite pouvant être la surreprésentation féminine de l'échantillon étudiant ramenée à la population générale ce qui, dans le cadre d'études sur "la dépression", peut avoir une conséquence non négligeable sur le taux de prévalence mesurée et contribuer également à expliquer la différence observée entre population étudiante et population générale, indépendamment de la spécificité du statut étudiant.

Le fait d'être une femme semble d'ailleurs être le facteur de risque de "dépression" le plus établi dans la littérature scientifique, mais les explications de cette co-occurence, certes répliquée dans de nombreuses études, demeurent complexes [22]. En effet plusieurs hypothèses concourantes peuvent être évoquées sur ce point. Tout d'abord, et c'est une des hypothèses fréquemment évoquées, nous ne savons pas dans quelle mesure des facteurs biologiques liés au sexe peuvent contribuer à expliquer cette association. Toutefois d'autres travaux nous incitent à la prudence sur des effets possibles d'une "naturalisation" des différences hommes/femmes concernant l'utilisation d'arguments biologiques comme explication principale voir exclusive de cette association. D'autres facteurs explicatifs comme les rôles sociaux et la complexité potentiellement plus grande pour les femmes de naviguer entre les paradoxes, injonctions et parfois des frustrations, injustices voir des violences subies qu'impliquent ces rôles sont, à juste titre, proposées. D'autres facteurs plus en rapport à des aspects tant de conceptualisation que de psychométrie sont moins souvent évoqués. Sur les aspects de conceptualisation, il a été proposé que la symptomatologie "dépressive" pourrait ne pas être considérée comme une entité unique applicable telle quelle à tout être humain mais différente selon les cultures. Il se pourrait également que la "dépression" puisse revêtir un aspect genré et donc présenter des formes symptomatologiques différentes voir spécifiques en fonction du genre, questionnant ainsi son "universalité". Concernant les aspects plus psychométriques, on ne sait pas par exemple, dans quelle mesure les hommes et les femmes considèrent de manière identique ou différente les questions qui leur sont posées, leurs réponses à ces questions et formulent la réponse donnée in fine. Ces aspects sont souvent évoqués concernant des méthodologies évaluant l'invariance de la mesure en fonction de groupes prédéfinis (par tranches d'âge, catégorie de sexe, groupes socio-démographiques, type de pathologies...) et notamment sur la question des seuils spécifique et potentiellement différenciés de réponses aux items ou questions entre groupes. Il est enfin envisageable que ces éléments de conceptualisation et de psychométrie puissent tout autant être influencés par des aspects biologiques que sociaux.

Au-delà de ces différentes questions et limites possibles que nous avons évoquées ainsi que de l'ouverture vers des modélisations plus complexes comme une des réponses possibles, la santé mentale n'en demeure pas moins importante. Comme nous l'avons rappelé, elle contribue à un coût élevé pour la société (plus de 100 milliards d'euros par an), majoritairement lié aux conséquences indirectes des troubles. Les étudiants constituent une population d'intérêt, car la plupart des troubles psychiatriques débutent à cet âge et qu'une action ou une absence de prévention pourront potentiellement engendrer un impact important sur le long terme.

\section{Conclusion}

Avant la crise du COVID, les rares données françaises indiquaient déjà une prévalence élevée de souffrance psychique chez les étudiants s'accompagnant d'un faible recours au soin par rapport à la population générale. Cette vision reste toutefois très générale en raison du manque de données et de comparabilité des études existantes. Les étudiants n'en demeurent pas moins une population importante du point de vue de la stratégie de prévention et d'intervention dans la mesure où la majorité des troubles psychiatriques semblent débuter ou ont déjà débuté à cette période de la vie. Si les actions de prévention peuvent donc avoir ici un impact important sur le long terme, un article rappel également le besoin de bien coordonner ces actions entre différents acteurs et sur différents niveaux [23] : « La prévention [...] s'est traditionnellement concentrée sur les personnes à risque, favorisant les compétences et les connaissances psychologiques au niveau individuel pour améliorer la capacité de surmonter les défis lorsqu'ils se produisent. [...] Ces approches fonctionnent en identifiant les individus à risque (comme dans les écoles) et entraînent donc un risque de stigmatisation. Souvent, ils ne tiennent pas suffisamment compte des besoins culturels et contextuels et sont limités dans l'intégration des facteurs de risques divers et distaux de dépression au-delà des compétences individuelles. De plus, il existe de multiples programmes, plates-formes de prestation et activités [...] ce qui risque de diffuser et de poser des obstacles subséquents à l'adoption et à la mise en cuvre à grande échelle. Il est important de noter que certaines stratégies sont devenues des programmes comportant plusieurs niveaux, favorisant les compétences individuelles ainsi que le soutien à l'intégration de la prévention, telles que l'intégration des politiques de santé mentale et de bien-être dans les écoles et les lieux de travail. Le développement de programmes à plusieurs niveaux est une avancée importante dans le domaine de la prévention de la dépression, car ils prennent en compte les multiples niveaux auxquels les risques de dépression opèrent. Ces programmes vont au-delà des aptitudes et compétences au niveau individuel pour incorporer l'environnement social plus large et les pratiques culturelles des milieux dans lesquels les individus peuvent exploiter ces aptitudes. Il est probable que sans ces conditions, les efforts de prévention au niveau individuel ne seraient probablement pas optimaux. » 
Le manque de données comme de politique de production d'indicateurs à différents niveaux est d'autant plus regrettable que la santé mentale continue, semble-t-il, de générer un coût économique (direct et indirect) très élevé et que les études montrent une bonne efficacité et rentabilité des actions de prévention et d'intervention précoce. La crise du COVID avec ses conséquences psychosociales risque d'aggraver les problèmes des jeunes adultes en général et des étudiants en particulier ${ }^{27}$. Dans une interview ${ }^{28}$ Patrick McGorry, l'un des spécialistes mondiaux de la prévention et de l'intervention précoce pointait la situation comme " une véritable discrimination entre santé mentale et santé somatique ». Il indiquait à ce titre sur la question de l'accès aux soins et aux traitements qu' " il y a tellement de jeunes qui ne reçoivent aucune aide. Le fait qu'on néglige de traiter ces jeunes a de véritables conséquences. Les critiques ont tendance à parler de surdiagnostic, mais - alors que pour certains jeunes, on peut parler de mauvais diagnostic et de traitement inapproprié- on est très loin de proposer trop de traitements. Le contraire est vrai : nous sommes confrontés à un problème considérable parce que les traitements sont insuffisants ou totalement absents ». L'ancien directeur du National Institute of Mental Health (NIMH) aux États-Unis, le Dr Insel, déclarait également dans un article du New-York Times ${ }^{29}$ que «c'est la qualité des soins et non la quantité qui compte " et que "nous avons besoin de plus d'accès, de meilleures mesures et d'une meilleure qualité des soins ».

Même si certains considèrent, à juste titre, que la solidité des connaissances en psychologie n'est peut-être pas encore suffisamment mature pour se prononcer sur les conséquences psychologiques à venir de la pandémie ou encore pour définir des politiques d'actions publiques [24], il n'en demeure pas moins important de tenter de mesurer ces phénomènes de la manière la plus transparente possible en s'appuyant sur une démarche de Science Ouverte ou Open Science. C'est pourquoi il apparait essentiel de mettre en place des systèmes d'évaluation de la santé mentale des étudiants au niveau national et local ainsi que des actions de prévention et de soins destinées à ces derniers sans pour autant réduire la complexité au seul statut étudiant pas plus que d'ignorer la complexité [25] du psychique, de sa mesure et de sa prise en charge.

\section{Déclaration de liens d'intérêts}

Yannick Morvan a reçu des financements de l'université Paris Nanterre, de l'Inserm, du GHU Paris Psychiatrie Neurosciences, de la Fondation de France, de la Fondation Pierre Deniker. Yannick Morvan est ou a été membre de différentes organisations professionnelles et scientifiques de ou impliquant des psychologues (AEPU, AFTCC, AFRC, APA, APS, IdPsy, IEPA, FFPP, SFP). Il est également membre du collège scientifique de l'Observatoire National de la Vie Etudiante (OVE) et du comité scientifique du congrès de l'Encéphale. Yannick Morvan a été sollicité comme expert par le cabinet Ernst \& Young. Ariel Frajerman a reçu des financements de Agence Nationale de la Recherche (ANR), ARS Ile-de-France.

\section{Remerciements}

Les auteurs tiennent à remercier l'Observatoire de la Vie Etudiante et notamment Monique Ronzeau, Elise Tenret, Feres Belghith et

\footnotetext{
$\overline{27}$ http://theconversation.com/

covid-19-quelles-consequences-sur-la-sante-mentale-137242

28 https://www.escap.eu/policy/access-to-mental-health-care/mcgorry-french

29 https://www.nytimes.com/2018/06/08/health/suicide-spade-bordain-cdc.html
}

Odile Ferry ainsi qu'à l'ensemble des collègues ayant contribué à l'enquête Santé de l'OVE et plus particulièrement à Boris Chaumette, Fabien Gierski et Doriane Mignon ; le réseau RESPPET et la Fondation Santé des Etudiants de France notamment Dominique Monchablon, Nathalie Godard, Christophe Ferveur, Catherine Brébant et Anne Delbende ; le réseau des services de médecine préventive universitaire et des médecins directeurs notamment Bernard Lelu, Joana Rouvier, Christophe Tzourio, Laurent Gerbaud et Martine Rosenbacher-Berlemont ; Jérôme Mourroux du Cabinet Ernst \& Young ; Yann Le Nevé ancien ingénieur au SHU de l'Hôpital Ste Anne et Astrid Chevance de l'équipe METHODS de l'Inserm; enfin toutes les personnes impliquées dans l'approche en réseau en psychologie et notamment les groupes psychosystem, psych networks, psychological dynamics ainsi que le psychological networks amsterdam summer school 2020

\section{Références}

[1] R.C. Kessler, P. Berglund, O. Demler, et al., Lifetime prevalence and age-of-onset distributions of DSM-IV disorders in the National Comorbidity Survey Replication, Arch Gen Psychiatry 62 (2005) 593-602.

[2] S. Dalsgaard, E. Thorsteinsson, B.B. Trabjerg, et al., Incidence rates and cumulative incidences of the full spectrum of diagnosed mental disorders in childhood and adolescence, JAMA Psychiatry (2019).

[3] P. Verger, V. Guagliardo, F. Gilbert, et al., Psychiatric disorders in students in six French universities: 12-month prevalence, comorbidity, impairment and help-seeking, Soc Psychiatry Psychiatr Epidemiol 45 (2010) 189-199.

[4] N. Reavley, A.F. Jorm, Prevention and early intervention to improve mental health in higher education students: a review, Early Interv Psychiatry 4 (2010) $132-142$.

[5] R. Kendell, A. Jablensky, Distinguishing between the validity and utility of psychiatric diagnoses, Am J Psychiatry 160 (2003) 4-12.

[6] C. Hammen, Risk factors for depression: an autobiographical review, Annu Rev Clin Psychol (2018) 14.

[7] S.D. Hollon, R.J. DeRubeis, P.W. Andrews, et al., Cognitive therapy in the treatment and prevention of depression: a fifty-year retrospective with an evolutionary coda, Cogn Ther Res (2020).

[8] C. Léon, C. Chan-Chee, E. du Roscoät, le groupe Baromètre de Santé publique France 2017. Baromètre de Santé publique France 2017: tentatives de suicide et pensées suicidaires chez les 18-75 ans, BEH (2019) 38-47.

[9] L. Boschloo, C.D. Borkulo, van, et al., The network structure of symptoms of the diagnostic and statistical manual of mental disorders, PLOS ONE 10 (2015) e 0137621

[10] D. Borsboom, A. Cramer, A. Kalis, Brain disorders ? Not really... Why network structures block reductionism in psychopathology research, Behav Brain Sci (2018) 1-54.

[11] G. Lunansky, C. Borkulo, D. Borsboom, Personality, resilience, and psychopathology: a model for the interaction between slow and fast network processes in the context of mental health, Eur J Personal (2020).

[12] D. Borsboom, H. van der Maas, J. Dalege, et al., Theory construction methodology: a practical framework for theory formation in psychology, PsyArXiv (2020).

[13] E.I. Fried, The 52 symptoms of major depression: Lack of content overlap among seven common depression scales, J Affect Disord (2017) https://doi.org/ 10.1016/j.jad.2016.10.019.

[14] E.I. Fried, F. Coomans, L. Lorenzo-Luaces, The 341737 ways of qualifying for the melancholic specifier, Lancet Psychiatry 7 (2020) 479-480.

[15] L.S. Rotenstein, M.A. Ramos, M. Torre, et al., Prevalence of depression, depressive symptoms, and suicidal ideation among medical students: a systematic review and meta-analysis, JAMA 316 (2016) 2214-2236.

[16] E.I. Fried, F. Papanikolaou, S. Epskamp, Mental health and social contact during the COVID-19 pandemic: an ecological momentary assessment study, PsyArXiv (2020) https://doi.org/10.31234/osf.io/36xkp.

[17] M. Taquet, J. Quoidbach, E.I. Fried, et al., Mood homeostasis before and during the Coronavirus disease 2019 (COVID-19) lockdown among students in the Netherlands, JAMA Psychiatry (2020) https://doi.org/10.1001/jamapsychiatry. 2020.2389.

[18] M. Pierce, S. McManus, C. Jessop, et al., Says who? The significance of sampling in mental health surveys during COVID-19, Lancet Psychiatry 7 (2020) 567-568.

[19] A. Saltelli, G. Bammer, I. Bruno, et al., Five ways to ensure that models serve society: a manifesto, Nature 582 (2020) 482-484. 
[20] A.M. Beltz, A.G.C. Wright, B.N. Sprague, et al., Bridging the nomothetic and idiographic approaches to the analysis of clinical data, Assessment 23 (2016) 447-458.

[21] P. Patalay, E.I. Fried, Editorial Perspective:, Prescribing measures: unintended negative consequences of mandating standardized mental health measurement, J Child Psychol Psychiatry (2020) https://doi.org/10.1111/jcpp.13333.

[22] C. Kuehner, Why is depression more common among women than among men?, Lancet Psychiatry 4 (2017) 146-158.
[23] E. Hoare, E. Callaly, M. Berk, Can depression be prevented?. If so how?, JAMA Psychiatry (2020) https://doi.org/10.1001/jamapsychiatry.2020.1273.

[24] H. IJzerman, N.A. Lewis, A.K. Przybylski, et al., Use caution when applying behavioural science to policy, Nat Hum Behav (2020) https://doi.org/10.1038/ s41562-020-00990-w.

[25] E.I. Fried, D.J. Robinaugh, Systems all the way down: embracing complexity in mental health research, BMC Med (2020) 18, https://doi.org/10.1186/s12916020-01668-w. 